\title{
A Highly Efficient and General Method for the Preparation of $(Z)$-Allylic Bromides Derived from Morita-Baylis-Hillman Adducts
}

\author{
Misael Ferreira, Luciano Fernandes and Marcus M. Sá* \\ Departamento de Química, Universidade Federal de Santa Catarina, 88040-900 Florianópolis-SC, Brazil
}

(Z)-2-(Bromometil)-2-alcenoatos são importantes intermediários sintéticos e foram preparados de forma simples e prática pelo tratamento de $\alpha$-metileno- $\beta$-hidroxiésteres (produtos da reação de Morita-Baylis-Hillman) com $\mathrm{LiBr} / \mathrm{H}_{2} \mathrm{SO}_{4}$ em acetonitrila à temperatura ambiente. Além de fornecer altos rendimentos e de tolerar a presença de diversos grupos funcionais, esta nova metodologia utiliza reagentes baratos e não faz uso de $\mathrm{HBr}$.

Representative (Z)-2-(bromomethyl)-2-alkenoates were easily prepared in high yield by treating $\alpha$-methylene- $\beta$-hydroxyesters (Morita-Baylis-Hillman adducts) with $\mathrm{LiBr} / \mathrm{H}_{2} \mathrm{SO}_{4}$ in acetonitrile at room temperature. Besides the tolerance to many diverse functional groups, this new methodology employs inexpensive reagents and avoids the use of $\mathrm{HBr}$.

Keywords: Morita-Baylis-Hillman reaction, $\alpha$-methylene- $\beta$-hydroxyesters, $(Z)$-allylic bromides, $N$-allyl acetamides

\section{Introduction}

Allylic bromides $\mathbf{1}$ are versatile building blocks of many important substances including natural products, heterocycles and biologically-active molecules ${ }^{1-13}$ (Scheme 1). These multifunctional compounds can be prepared from $\alpha$-methylene- $\beta$-hydroxyesters 2 , which are easily obtained by the well-recognized Morita-BaylisHillman reaction. ${ }^{14-17}$

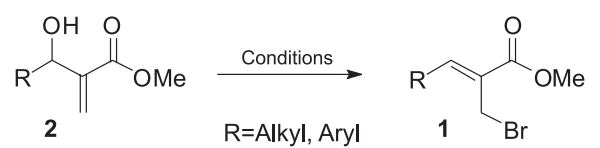

Scheme 1

Typically, the method of choice for the direct conversion of adduct $\mathbf{2}$ to the corresponding (Z)-allylic bromide 1 relies on the combination of $48 \% \mathrm{HBr}$ and conc. $\mathrm{H}_{2} \mathrm{SO}_{4}$ in $\mathrm{CH}_{2} \mathrm{Cl}_{2}$ at low temperature. ${ }^{18}$ In spite of the fairly good yields often achieved for this transformation, the required use of large quantities of noxious hydrobromic acid is a major disadvantage due to safety and environmental concerns. Alternative methods for converting alcohol 2 to bromide 1 include the use of $\mathrm{NBS} / \mathrm{Me}_{2} \mathrm{~S},{ }^{12} \mathrm{PBr}_{3},{ }^{19} \mathrm{Br}\left(\mathrm{Me}_{2}\right) \mathrm{S}^{+} \mathrm{Br}^{-},{ }^{20}$

*e-mail: msa@qmc.ufsc.br and also bromine salts immobilized in ionic liquids ${ }^{21}$ or in the presence of solid catalysts such as montmorillonite KSF clay, ${ }^{22}$ Amberlyst- $15,{ }^{23}$ silica gel-supported $\mathrm{NaHSO}_{4},{ }^{24}$ and silica gel alone under microwave irradiation. ${ }^{25}$ Although all the above procedures have their own applications, many of them suffer from one or more drawbacks such as the use of harmful conditions, high temperatures, special equipment or commercially unavailable reagents or catalysts, eventually leading to long reaction times or low yields. Consequently, there is a great need to develop a simple and general method for the synthesis of allylic bromides $\mathbf{1}$.

In a continuation of our work on the Morita-BaylisHilllman reaction, ${ }^{26-29}$ herein we present a straightforward methodology for high-yield preparation of $(Z)$-allylic bromides 1 using $\mathrm{LiBr} / \mathrm{H}_{2} \mathrm{SO}_{4}$ in acetonitrile at room temperature.

\section{Results and Discussion}

$\alpha$-Methylene- $\beta$-hydroxyester $\mathbf{2} \mathbf{a}^{30}$ was submitted to a variety of reaction conditions in order to achieve optimal conversion to allylic bromide 1a. Initially, treatment of alcohol $2 \mathbf{a}$ with conc. $\mathrm{HBr}(48 \%$, from a freshly-opened bottle) in acetonitrile or dichloromethane during $24 \mathrm{~h}$ led only to negligible yields $(<20 \%)$ of $(Z)$-allylic bromide 1a. On the other hand, a combination of sulfuric acid and 
lithium bromide in acetonitrile as the solvent showed promising results even with nearly stoichiometrically amounts of reagents (Table 1, entry 1). Increasing the amount of either sulfuric acid or lithium bromide led to an improvement in the reaction rate (entries 2 and 3), but a two-fold excess of both the acid and the bromine salt caused a slight diminution in the conversion (entry 4). After some experimentation we found that an optimized ratio of 2.0 equiv. $\mathrm{LiBr}$ to 2.5 equiv. $\mathrm{H}_{2} \mathrm{SO}_{4}$ resulted in an excellent conversion to $(Z)$-allylic bromide $\mathbf{1 a}$ after a short time (Table 1, entry 5).

Reaction rates were also strongly influenced by the nature of the solvent (or solvent combination) used, with acetonitrile being highly superior to acetone, THF and aqueous combinations (Table 1, entries 6-9). The strength of the acid required to mediate the transformation was evaluated in control reactions by replacement of sulfuric acid with phosphoric acid or para-toluenesulfonic acid (TsOH). In spite of the moderate conversion observed for $\mathrm{TsOH}$ after a reasonable period (Table 1, entry 10), the result with $\mathrm{H}_{3} \mathrm{PO}_{4}$ was unsatisfactory (entry 11).

Finally, a competitive reaction took place when $\mathrm{NaBr}$ and $\mathrm{KBr}$ were tested as the source of bromide (Table 1, entries 12 and 13). Besides the lower conversions to $\mathbf{1 a}$, the starting alcohol 2a was totally consumed to generate an inseparable mixture of isomeric $N$-allyl acetamides $\mathbf{3 / 4}$ in a 1.5:1 ratio (Scheme 2). The competitive formation of allylic acetamides $\mathbf{3 / 4}$ was confirmed by running the reaction in the absence of any bromine salt, which resulted in the expected 1.5:1 mixture of acetamides $\mathbf{3 / 4}$ (Table 1, entry 14). Participation of adduct 2 in this Ritter-type reaction has been recently described using either sulfonic acid or Amberlist-15 under reflux for hours, ${ }^{31,32}$ but in our case the acetamide $\mathbf{3}$ was obtained at ambient temperature after a few minutes as a mixture with the unexpected isomer 4. Unfortunately, attempts to separate the isomers by chromatography were unsuccessful.

The best condition found for converting $\alpha$-methylene$\beta$-hydroxyester 2a into (Z)-allylic bromide 1a (Table 2 , entry 1) was further extended to other representative Morita-Baylis-Hillman adducts 2. As can be seen from data in Table 2, good to excellent yields were obtained in all cases regardless the substitution pattern on the starting $\alpha$-methylene- $\beta$-hydroxyester 2 . However, a distinct difference in reactivity was clearly detected. Substrates containing electron-donating groups were the most reactive under Condition A (2.0 equiv. $\mathrm{LiBr}, 2.5$ equiv. $\mathrm{H}_{2} \mathrm{SO}_{4}$ ) and gave the corresponding bromides $\mathbf{1 b}$-e quantitatively after a few minutes (Table 2, entries 2-5). This high reactivity allowed a reduction in the amount of $\mathrm{LiBr}$ to nearly 1 equiv. without compromising the reaction rate or yield (results not shown).

Table 1. Conversion of $\alpha$-methylene- $\beta$-hydroxyester 2a to (Z)-allylic bromide 1a under different conditions

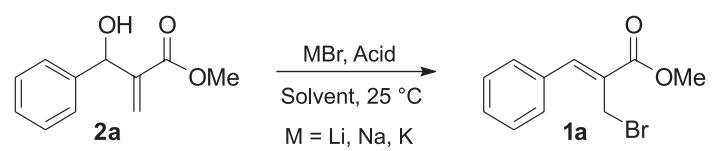

\begin{tabular}{|c|c|c|c|c|c|}
\hline Entry & Salt (equiv.) & Acid (equiv.) & Solvent system & time $/ \mathrm{h}$ & Conversion to $\mathbf{1 a} /(\%)^{\mathrm{a}}$ \\
\hline 1 & $\operatorname{LiBr}(1.1)$ & $\mathrm{H}_{2} \mathrm{SO}_{4}(1.0)$ & $\mathrm{CH}_{3} \mathrm{CN}$ & 1 & 66 \\
\hline 2 & $\operatorname{LiBr}(2.0)$ & $\mathrm{H}_{2} \mathrm{SO}_{4}(1.0)$ & $\mathrm{CH}_{3} \mathrm{CN}$ & 1 & 78 \\
\hline 3 & $\operatorname{LiBr}(1.1)$ & $\mathrm{H}_{2} \mathrm{SO}_{4}(2.0)$ & $\mathrm{CH}_{3} \mathrm{CN}$ & 1 & 90 \\
\hline 4 & $\operatorname{LiBr}(2.0)$ & $\mathrm{H}_{2} \mathrm{SO}_{4}(2.0)$ & $\mathrm{CH}_{3} \mathrm{CN}$ & 1 & 85 \\
\hline 5 & $\operatorname{LiBr}(2.0)$ & $\mathrm{H}_{2} \mathrm{SO}_{4}(2.5)$ & $\mathrm{CH}_{3} \mathrm{CN}$ & 1 & 100 \\
\hline 6 & $\operatorname{LiBr}(2.0)$ & $\mathrm{H}_{2} \mathrm{SO}_{4}(2.5)$ & Acetone & 1.5 & 50 \\
\hline 7 & $\operatorname{LiBr}(2.0)$ & $\mathrm{H}_{2} \mathrm{SO}_{4}(2.5)$ & THF & 1.5 & 5 \\
\hline 8 & $\operatorname{LiBr}(2.0)$ & $\mathrm{H}_{2} \mathrm{SO}_{4}(2.5)$ & $\mathrm{CH}_{3} \mathrm{CN} / \mathrm{H}_{2} \mathrm{O}(3: 1)$ & 1.5 & 0 \\
\hline 9 & $\operatorname{LiBr}(2.0)$ & $\mathrm{H}_{2} \mathrm{SO}_{4}(2.5)$ & Acetone $/ \mathrm{H}_{2} \mathrm{O}(3: 1)$ & 1.5 & 0 \\
\hline 10 & $\operatorname{LiBr}(2.0)$ & $\mathrm{TsOH}(2.0)$ & $\mathrm{CH}_{3} \mathrm{CN}$ & 7 & 70 \\
\hline 11 & $\operatorname{LiBr}(2.0)$ & $\mathrm{H}_{3} \mathrm{PO}_{4}(2.5)$ & $\mathrm{CH}_{3} \mathrm{CN}$ & 24 & 10 \\
\hline 12 & $\mathrm{NaBr}(2.0)$ & $\mathrm{H}_{2} \mathrm{SO}_{4}(2.5)$ & $\mathrm{CH}_{3} \mathrm{CN}$ & 1 & $75[25]^{\mathrm{b}}$ \\
\hline 13 & $\mathrm{KBr}(2.0)$ & $\mathrm{H}_{2} \mathrm{SO}_{4}(2.5)$ & $\mathrm{CH}_{3} \mathrm{CN}$ & 5 & $38[62]^{\mathrm{b}}$ \\
\hline 14 & - & $\mathrm{H}_{2} \mathrm{SO}_{4}(2.5)$ & $\mathrm{CH}_{3} \mathrm{CN}$ & 0.2 & $0[100]^{\mathrm{b}}$ \\
\hline
\end{tabular}

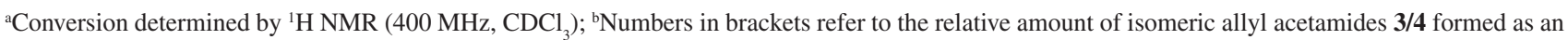
inseparable mixture (1.5:1 ratio). 


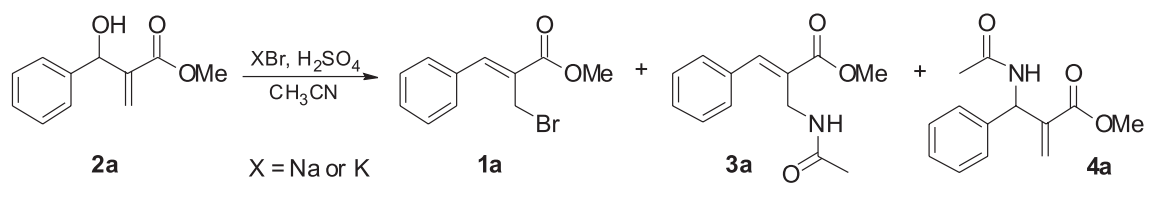

Scheme 2

Table 2. Synthesis of (Z)-allylic bromides 1 from alcohols 2 using $\mathrm{LiBr} / \mathrm{H}_{2} \mathrm{SO}_{4}$ in acetonitrile

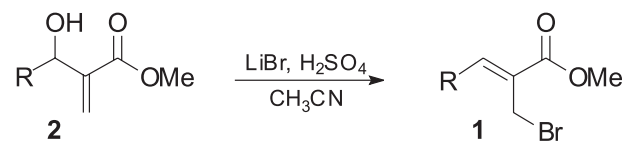

\begin{tabular}{|c|c|c|c|c|c|}
\hline Entry & Allylic bromide & $\mathrm{R}$ & Condition $^{\mathrm{a}}$ & time / h & Yield / $(\%)^{\mathrm{b}}$ \\
\hline 1 & $1 \mathrm{a}$ & $\mathrm{C}_{6} \mathrm{H}_{5}$ & A & 1 & 90 \\
\hline 2 & $1 b$ & 2- $\mathrm{C}_{10} \mathrm{H}_{7}$ & A & 0.5 & 95 \\
\hline 3 & $1 \mathrm{c}$ & 4- $\mathrm{CH}_{3} \mathrm{OC}_{6} \mathrm{H}_{4}$ & A & 0.5 & 91 \\
\hline 4 & 1d & $3,4-\left(\mathrm{CH}_{3} \mathrm{O}\right)_{2} \mathrm{C}_{6} \mathrm{H}_{3}$ & A & 0.5 & 95 \\
\hline 5 & $1 e$ & $3,4-\left(\mathrm{OCH}_{2} \mathrm{O}\right) \mathrm{C}_{6} \mathrm{H}_{3}$ & A & 0.5 & 87 \\
\hline 6 & 1f & $4-\mathrm{ClC}_{6} \mathrm{H}_{4}$ & A & 2 & 91 \\
\hline 7 & $1 \mathrm{~g}$ & $2-\mathrm{ClC}_{6} \mathrm{H}_{4}$ & A & 4 & 80 \\
\hline 8 & $1 \mathrm{~g}$ & & B & 3 & 89 \\
\hline 9 & $1 \mathrm{~h}$ & $2,4-\mathrm{ClC}_{6} \mathrm{H}_{3}$ & A & 24 & 88 \\
\hline 10 & $1 \mathrm{~h}$ & & B & 3 & 91 \\
\hline 11 & $\mathbf{1 i}$ & $4-\mathrm{NO}_{2} \mathrm{C}_{6} \mathrm{H}_{4}$ & A & 24 & 82 \\
\hline 12 & $1 \mathbf{i}$ & & B & 5 & 87 \\
\hline 13 & $\mathbf{1 j}$ & $3-\mathrm{NO}_{2} \mathrm{C}_{6} \mathrm{H}_{4}$ & B & 5 & 75 \\
\hline 14 & $1 \mathrm{k}$ & $\mathrm{CH}_{3}$ & A & 1 & 85 \\
\hline 15 & 11 & $\mathrm{CH}_{3} \mathrm{CH}_{2}$ & A & 1 & 76 \\
\hline
\end{tabular}

${ }^{a}$ Condition A: 2.0 equiv. $\mathrm{LiBr}, 2.5$ equiv. $\mathrm{H}_{2} \mathrm{SO}_{4}$, acetonitrile, $25{ }^{\circ} \mathrm{C}$. Condition B: 4.0 equiv. $\mathrm{LiBr}, 5.0$ equiv. $\mathrm{H}_{2} \mathrm{SO}_{4}$, acetonitrile, $25^{\circ} \mathrm{C}$; ${ }^{\mathrm{b}} \mathrm{Isolated}$ yields after purification by column chromatography.

On the other hand, chloro-substituted derivatives were less reactive and acceptable yields were only obtained after a prolonged time at room temperature (Table 2, entries 7 and 9). In an effort to improve the conversion rate by using higher amounts of reagents, $\mathbf{2} \mathbf{g}$ and $\mathbf{2 h}$ were treated with 4.0 equiv. $\mathrm{LiBr}$ and 5.0 equiv. $\mathrm{H}_{2} \mathrm{SO}_{4}$ (Condition $\mathrm{B}$ ). Indeed, this more extreme condition led to the corresponding bromides $1 \mathrm{~g}$ and $\mathbf{1 h}$ in better yields after shorter periods (entries 8 and 10). As expected, nitro-substituted adducts $\mathbf{2 i}, \mathbf{j}$ were the most unreactive substrates under Condition A (entry 11), but good yields in reasonable time were achieved with Condition B (entries 12 and 13). Finally, alkylsubstituted substrates $\mathbf{2 k}$,I were also successful transformed in the corresponding bromides $\mathbf{1 k}, \mathbf{l}$ by employing Condition A (entries 14 and 15), thus demonstrating the generality of the method.

The anticipated $Z$-stereochemistry assigned to all allylic bromides 1 was based on the characteristic NMR shift of the highly deshielded $\beta$-olefinic hydrogen cis to the carboxyl group ${ }^{15,18,22-24}$ and also on the previous X-ray crystallography analysis of $\mathbf{1 e} .^{33}$ Products were obtained nearly pure and no signals for an elusive formation of $E$-isomer was ever detected.

\section{Conclusions}

An efficient methodology to access representative ( $Z$ )2-(bromomethyl)-2-alkenoates 1 based on readily available reagents and simple experimental conditions has been developed. The high yields and excellent $Z$-stereoselectivity observed in all cases, even for substrates carrying electronwithdrawing groups, makes this method very attractive and expands the potential applicability of allylic bromides in synthesis. Mechanistic studies dealing with the formation of $\mathbf{1}$ and their use in synthetic transformations are currently under investigation. 


\section{Experimental}

\section{General procedures}

All chemicals were of reagent grade (Aldrich, CarloErba, JTBaker, Nuclear, Riedel, Strem) and were used as received. Melting points were determined using a Microquímica MQPF301 apparatus and are uncorrected. Infrared spectra were acquired with a Perkin-Elmer FTIR 1600 spectrometer using $\mathrm{KBr}$ for solids and film for liquid samples (range 4000-400 $\mathrm{cm}^{-1}$ ). ${ }^{1} \mathrm{H}$ NMR (400 MHz) and ${ }^{13} \mathrm{C}$ NMR (100 MHz, fully decoupled) spectra were recorded with a Varian AS-400 spectrometer. Samples were prepared in $\mathrm{CDCl}_{3}$ solution containing 1-2\% tetramethylsilane (TMS) as internal standard. Chemical shifts are reported in parts per million $(\delta)$ relative to TMS. Elemental analyses were conducted in Carlo Erba CHNS EA-1110 equipment by UFSC-Central Analítica, Departamento de Química, Florianópolis, SC, Brazil. Purifications by column chromatography were performed with silica gel (Aldrich, 100-200 mesh particle size). Compounds $\mathbf{2 a}, \mathbf{b}, \mathbf{c}, \mathbf{e}, \mathbf{f}, \mathbf{h}, \mathbf{i}, \mathbf{j}, \mathbf{k}, \mathbf{l}$ were prepared according to the known methods and showed physical and spectral data in accordance with their expected structure and by comparison with data in literature. ${ }^{11,29,30,33-36}$

Methyl 3-hydroxy-2-methylene-3-(3,4-dimethoxyphenyl) propanoate $(\mathbf{2 d})$

Clear yellow oil; IR (neat) $v_{\max } / \mathrm{cm}^{-1}: 3496,3000,2949$, 2839, 1719, 1631, 1512, 1444, 1262, 1145, 1032; ${ }^{1} \mathrm{H}$ NMR: $\delta 3.65(\mathrm{~s}, 3 \mathrm{H}), 3.80(\mathrm{~s}, 6 \mathrm{H}), 5.45(\mathrm{~s}, 1 \mathrm{H}), 5.81(\mathrm{~s}, 1 \mathrm{H}), 6.26$ $(\mathrm{s}, 1 \mathrm{H}), 6.76(\mathrm{~d}, J 8.0 \mathrm{~Hz}, 1 \mathrm{H}), 6.82(\mathrm{~d}, J 8.0 \mathrm{~Hz}, 1 \mathrm{H}), 6.86$ (s, 1H); ${ }^{13} \mathrm{C}$ NMR: $\delta 52.1\left(\mathrm{CH}_{3}\right), 56.0\left(2 \mathrm{x} \mathrm{CH}_{3}\right), 72.9(\mathrm{CH})$, $110.1(\mathrm{CH}), 111.1(\mathrm{CH}), 119.2(\mathrm{CH}), 125.8\left(=\mathrm{CH}_{2}\right), 134.2$ (C), 142.4 (C), 148.8 (C), 149.1 (C), $167.1(\mathrm{C}=\mathrm{O})$.

Methyl 3-hydroxy-2-methylene-3-(2-chlorophenyl) propanoate $(\mathbf{2 g})$

Colorless oil; IR (neat) $v_{\max } / \mathrm{cm}^{-1}: 3437,3067,3000$, 2953, 1720, 1632, 1440, 1270, 1149, 1032; ${ }^{1} \mathrm{H}$ NMR: $\delta$ 3.74 (s, 3H), 5.58 (s, 1H), 5.95 (s, 1H), 6.31 (s, 1H), 7.19$7.34(\mathrm{~m}, 3 \mathrm{H}), 7.53(\mathrm{dd}, J 1.5,6.0 \mathrm{~Hz}, 1 \mathrm{H}),{ }^{13} \mathrm{C} \mathrm{NMR}: \delta$ $52.4\left(\mathrm{CH}_{3}\right), 69.4(\mathrm{CH}), 127.2\left(=\mathrm{CH}_{2}\right), 127.3(\mathrm{CH}), 128.4$ $(\mathrm{CH}), 129.2(\mathrm{CH}), 129.7(\mathrm{CH}), 133.0(\mathrm{C}), 138.6(\mathrm{C}), 140.9$ (C), $167.2(\mathrm{C}=\mathrm{O})$.

Typical procedure for the synthesis of (Z)-2-(bromomethyl)2-alkenoates (1)

To a stirred solution of a Morita-Baylis-Hillman adduct $2(5.0 \mathrm{mmol})$ in $15.0 \mathrm{~mL}$ of acetonitrile at $0-5{ }^{\circ} \mathrm{C}$ were added $\mathrm{LiBr}$ (Condition A: $10.0 \mathrm{mmol}$; Condition B: $20.0 \mathrm{mmol}$ ) and $96 \% \mathrm{H}_{2} \mathrm{SO}_{4}$ (Condition A: $12.5 \mathrm{mmol}$; Condition B: $25.0 \mathrm{mmol}$ ). The reaction was allowed to warm up and stirring was continued at $25^{\circ} \mathrm{C}$ for the time presented in Table 2. The final mixture was diluted with $\mathrm{CH}_{2} \mathrm{Cl}_{2}$, and the organic extract was washed with $\mathrm{H}_{2} \mathrm{O}$, sat. $\mathrm{NaHCO}_{3}$ and brine, dried over $\mathrm{Na}_{2} \mathrm{SO}_{4}$, filtered and concentrated under reduced pressure. The resulting residue was purified by column chromatography (hexane/ethyl acetate 9:1) to give the corresponding allylic bromides $\mathbf{1}$. Compounds $\mathbf{1 a}, \mathbf{b}, \mathbf{e}, \mathbf{f}, \mathbf{g}, \mathbf{i}, \mathbf{k}, \mathbf{l}$ showed physical and spectral data in accordance with their expected structure and by comparison with data in literature..$^{8,11,18,20-25}$

Methyl (Z)-2-(bromomethyl)-3-(4-methoxyphenyl)-2propenoate $(\mathbf{1 c})$

White solid; mp 59.6-60.1 ${ }^{\circ} \mathrm{C}$; IR (KBr) $v_{\max } / \mathrm{cm}^{-1}: 3451$, 2936, 2836, 1705, 1596, 1507, 1439, 1171, 767; ${ }^{1} \mathrm{H}$ NMR: $\delta 3.86(\mathrm{~s}, 3 \mathrm{H}), 3.87(\mathrm{~s}, 3 \mathrm{H}), 4.45(\mathrm{~s}, 2 \mathrm{H}), 6.99(\mathrm{~d}, J 8.5 \mathrm{~Hz}$, 2H), 7.58 (d, J 8.5 Hz, 2H), $7.78(\mathrm{~s}, 1 \mathrm{H}) ;{ }^{13} \mathrm{C} \mathrm{NMR:} \delta 27.9$ $\left(\mathrm{CH}_{2}\right), 52.7\left(\mathrm{CH}_{3}\right), 55.7\left(\mathrm{CH}_{3}\right), 114.8(2 \times \mathrm{CH}), 126.5(\mathrm{C})$, 127.1 (C), 132.3 (2 x CH), 143.3 (=CH), 161.2 (C), 167.3 $(\mathrm{C}=\mathrm{O})$. Anal. Calc. for $\mathrm{C}_{12} \mathrm{H}_{13} \mathrm{BrO}_{3}(\%)$ : C, 50.55; $\mathrm{H}, 4.60$. Found: C, 50.80; H, 4.60.

Methyl (Z)-2-(bromomethyl)-3-(3,4-dimethoxyphenyl)-2propenoate $(\mathbf{l d})$

White solid; mp 79.1-79.4 ${ }^{\circ} \mathrm{C}$; IR (KBr) $v_{\max } / \mathrm{cm}^{-1}: 3455$, 2957, 2832, 1708, 1604, 1509, 1431, 1263, 768; ${ }^{1} \mathrm{H}$ NMR: $\delta 3.85(\mathrm{~s}, 3 \mathrm{H}), 3.91(\mathrm{~s}, 3 \mathrm{H}), 3.92(\mathrm{~s}, 3 \mathrm{H}), 4.44(\mathrm{~s}, 2 \mathrm{H}), 6.92$ $(\mathrm{d}, J 8.5 \mathrm{~Hz}, 1 \mathrm{H}), 7.16$ (dd, $J 2.0,8.5 \mathrm{~Hz}, 1 \mathrm{H}), 7.23$ (d, $J$ $2.0 \mathrm{~Hz}, 1 \mathrm{H}), 7.77(\mathrm{~s}, 1 \mathrm{H}) ;{ }^{13} \mathrm{C}$ NMR: $\delta 28.1\left(\mathrm{CH}_{2}\right), 52.7$ $\left(\mathrm{CH}_{3}\right), 56.2\left(\mathrm{CH}_{3}\right), 56.3\left(\mathrm{CH}_{3}\right), 111.4(\mathrm{CH}), 112.7(\mathrm{CH})$, 124.4 (CH), $126.4(\mathrm{C}), 127.2(\mathrm{C}), 143.6(=\mathrm{CH}), 149.2(\mathrm{C})$, $150.6(\mathrm{C}), 167.1(\mathrm{C}=\mathrm{O})$. Anal. Calc. for $\mathrm{C}_{13} \mathrm{H}_{15} \mathrm{BrO}_{4}(\%)$ : C, 49.54; H, 4.80. Found: C, 49.78; H, 5.12.

Methyl (Z)-2-(bromomethyl)-3-(2,4-dichlorophenyl)-2propenoate $(\mathbf{1 h})$

White solid; mp 75.4-75.7 ${ }^{\circ} \mathrm{C}$; IR (KBr) $v_{\max } / \mathrm{cm}^{-1}: 3416$, 3081, 1712, 1616, 1572, 1436, 1280, 765; ${ }^{1} \mathrm{H}$ NMR: $\delta 3.91$ (s, 3H), 4.24 (s, 2H), 7.37 (dd, J 2.2, 8.5 Hz, 1H), 7.47 (d, J $2.2 \mathrm{~Hz}, 1 \mathrm{H}), 7.66(\mathrm{~d}, J 8.5 \mathrm{~Hz}, 1 \mathrm{H}), 7.85(\mathrm{~s}, 1 \mathrm{H}) ;{ }^{13} \mathrm{C} \mathrm{NMR}$ : $\delta 26.2\left(\mathrm{CH}_{2}\right), 53.0\left(\mathrm{CH}_{3}\right), 127.8(\mathrm{CH}), 130.1(\mathrm{CH}), 130.7$ (CH), 131.3 (C), 131.7 (C), 135.7 (C), 136.3 (C), 138.6 $(=\mathrm{CH}), 166.2(\mathrm{C}=\mathrm{O})$. Anal. Calc. for $\mathrm{C}_{11} \mathrm{H}_{9} \mathrm{BrCl}_{2} \mathrm{O}_{2}(\%)$ : C, 40.78; H, 2.80. Found: C, 40.88; H, 2.84.

Methyl (Z)-2-(bromomethyl)-3-(3-nitrophenyl)-2propenoate $(\mathbf{1 j})$

Pale yellow solid; $\mathrm{mp} 80.5-82.0^{\circ} \mathrm{C}$; IR $(\mathrm{KBr}) \mathrm{v}_{\max } / \mathrm{cm}^{-1}$ : 
3394, 3077, 2952, 1709, 1624, 1532, 1432, 1355, 1299 1259, 772; ${ }^{1} \mathrm{H}$ NMR: $\delta 3.92(\mathrm{~s}, 3 \mathrm{H}), 4.33(\mathrm{~s}, 2 \mathrm{H}), 7.68(\mathrm{t}$, $J 7.5 \mathrm{~Hz}, 1 \mathrm{H}), 7.84(\mathrm{~s}, 1 \mathrm{H}), 7.92(\mathrm{~d}, J 7.5 \mathrm{~Hz}, 1 \mathrm{H}), 8.27$ $(\mathrm{d}, J 7.5 \mathrm{~Hz}, 1 \mathrm{H}), 8.42(\mathrm{~s}, 1 \mathrm{H}) ;{ }^{13} \mathrm{C} \mathrm{NMR}: \delta 25.5\left(\mathrm{CH}_{2}\right)$, $53.1\left(\mathrm{CH}_{3}\right), 124.4(\mathrm{CH}), 124.6(\mathrm{CH}), 130.4(\mathrm{CH}), 131.8$ (C), 135.2 (CH), 136.1 (C), 140.1 (=CH), 148.9 (C), 166.2 $(\mathrm{C}=\mathrm{O})$. Anal. Calc. for $\mathrm{C}_{11} \mathrm{H}_{10} \mathrm{BrNO}_{4}(\%)$ : C, 44.02; H, 3.36; N, 4.67. Found: C, 44.06; H, 3.25; N, 4.37.

\section{Acknowledgments}

The authors wish to thank Central de Análises (Departamento de Química, UFSC, Florianópolis) for spectroscopic analysis. M.M.S. is grateful to CNPq for a research fellowship. M.F and L.F. are grateful to CAPES and $\mathrm{CNPq}$ for fellowships. Financial support by PRONEX2003 (CNPq/FAPESC) and FAPESC (Santa Catarina State Research Council, Brazil, CON 16060/2007-1) is also gratefully acknowledged.

\section{References}

1. Deng, J.; Hu, X.-P.; Huang, J.-D.; Yu, S.-B.; Wang, D.-Y.; Duan, Z.-C.; Zheng, Z.; J. Org. Chem. 2008, 73, 2015.

2. Shanmugam, P.; Viswambharan, B.; Selvakumar, K.; Madhavan, S.; Tetrahedron Lett. 2008, 49, 2611.

3. Bakthadoss, M.; Sivakumar, N.; Sivakumar, G.; Murugan, G.; Tetrahedron Lett. 2008, 49, 829.

4. Rajan, Y. C.; Kanakam, C. C.; Selvam, S. P.; Murugesan, K.; Tetrahedron Lett. 2007, 48, 8562.

5. Lee, K. Y.; Gowrisankar, S.; Lee, Y. J.; Kim, J. N.; Tetrahedron 2006, 62, 8798 .

6. Lee, K. Y.; Kim, S. C.; Kim, J. N.; Tetrahedron Lett. 2006, 47, 977.

7. Chen, H.-Y.; Patkar, L. N.; Ueng, S.-H.; Lin, C.-C.; Lee, A. S.-Y.; Synlett 2005, 2035.

8. Fernandes, L.; Bortoluzzi, A. J.; Sá, M. M.; Tetrahedron 2004. 60, 9983.

9. Basavaiah, D.; Bakthadoss, M.; Pandiaraju, S.; Chem. Commun. 1998, 1639.

10. Basavaiah, D.; Hyma, R. S.; Tetrahedron 1996, 52, 1253.

11. Roush, W. R.; Brown, B. B.; J. Org. Chem. 1993, 58, 2151.

12. Hoffmann, H. M. R.; Rabe, J.; J. Org. Chem. 1985, 50, 3846.

13. Hoffmann, H. M. R.; Rabe, J.; Angew. Chem., Int. Ed. 1983, 22,795 .
14. Singh, V.; Batra, S.; Tetrahedron 2008, 64, 4511.

15. Basavaiah, D.; Rao, A. J.; Satyanarayana, T.; Chem. Rev. 2003, 103, 811 .

16. Ciganek, E.; Organic Reactions; Jonh Wiley \& Sons: New York, 1997, Vol. 51, pp. 201-350.

17. Lopes, E. C. S.; Coelho, F.; J. Braz. Chem. Soc. 2007, 18, 1415; Coelho, F.; Almeida, W. P.; Quim. Nova 2000, 23, 98.

18. Buchholz, R.; Hoffmann, H. M. R.; Helv. Chim. Acta 1991, 74, 1213.

19. Beltaïef, I.; Hbaïeb, S.; Besbes, R.; Amri, H.; Villiéras, M.; Villiéras, J.; Synthesis 1998, 1765.

20. Das, B.; Venkateswarlu, K.; Krishnaiah, M.; Holla, H.; Majhi, A.; Helv. Chim. Acta 2006, 89, 1417.

21. Ying, T. K.; Bao, W. L.; Wang, Z. H.; Zhang, Y. M.; J. Chem. Res. (S) 2005, 96.

22. Yadav, J. S.; Reddy, B. V. S.; Madan, C.; New J. Chem. 2001, 25,1114

23. Sá, M. M.; Ramos, M. D.; Fernandes, L.; Tetrahedron 2006, 62,11652 .

24. Das, B.; Banerjee, J.; Ravindranath, N.; Tetrahedron 2004, 60, 8357.

25. Gruiec, A.; Foucaud, A.; New J. Chem. 1991, 15, 943.

26. Sá, M. M.; Ferreira, M.; Fernandes, L.; Bortoluzzi, A. J.; Tetrahedron Lett. 2008, 49, 1228.

27. Sá, M. M.; Meier, L.; Fernandes, L.; Pergher, S. B. C.; Catal. Commun. 2007, 8, 1625.

28. Sá, M. M.; J. Braz. Chem. Soc. 2003, 14, 1005.

29. Nascimento, M. G.; Zanotto, S. P.; Melegari, S. P.; Fernandes, L.; Sá, M. M.; Tetrahedron: Asymmetry 2003, 14, 3111.

30. Foucaud, A.; Guemmout, F. E.; Bull. Soc. Chim. Fr. 1989, 403.

31. Hoen, R.; Tiemersma-Wegman, T.; Procuranti, B.; Lefort, L.; de Vries, J. G.; Minnaard, A. J.; Feringa, B. L.; Org. Biomol. Chem. 2007, 5, 267.

32. Das, B.; Majhi, A.; Banerjee, J.; Chowdhury, N.; J. Mol. Catal. A: Chem. 2006, 260, 32.

33. Bortoluzzi, A. J.; Fernandes, L.; Sá, M. M.; Acta Crystallogr., Sect. E: Struct. Rep. Online 2006, 62, o3391.

34. Yu, C.; Liu, B.; Hu, L.; J. Org. Chem. 2001, 66, 5413.

35. Cai, J.; Zhou, Z.; Zhao, G.; Tang, C.; Org. Lett. 2002, 4, 4723.

36. Coelho, F.; Almeida, W. P.; Veronese, D.; Mateus, C. R.; Lopes, E. C. S.; Rossi, R. C.; Silveira, G. P. C.; Pavam, C. H.; Tetrahedron 2002, 58, 7437. 